\title{
China White: Clinical Insights of an Evolving Designer Underground Drug
}

\author{
Carlos Ramos-Matos ${ }^{1}$, Wilfredo López-Ojeda ${ }^{2, *}$ \\ ${ }^{1}$ Department of Internal Medicine, University of South Florida, Morsani College of Medicine, USA \\ ${ }^{2}$ Department of Molecular Biology and Microbiology, University of Central Florida, College of Medicine, USA
}

Copyright (C) 2015 Horizon Research Publishing All rights reserved.

\begin{abstract}
Clandestine drug production, use, and exploitation present a social issue afflicting millions across the globe. "Designer opioids," some known to the street as "China White" for their alleged purity, have been unveiled in the past years; noted to possess properties that can compromise well-being in a remarkably novel way. This class of designer opioids, the 4-phenylpiperidines, seems to be generating significant medical concerns due to its deadly clinical manifestations; which may be further complicated by the ease of access and its potent addictive properties. The potential drug adulteration with other chemical formulations further complicates the medical scenario, posing a serious challenge in the management of presumed overdoses. Furthermore, if the key clinical manifestations are overlooked due to the presence confounding signs and symptoms to medical practitioners, patient's mortality may potentially increase even stronger. Thus, the purpose of this communication is to create awareness about these novel agents and their potentially devastating clinical complications. We strongly support the empiric treatment with naloxone per the currently established guidelines. In addition, we urge practitioners to carefully document findings relating to recovery time and historical data to aid in early detection, and precise decision-making in suspected intoxications cases.
\end{abstract}

Keywords Designer drugs, Opioids, Naloxone, Addiction

\section{Introduction}

Clandestine drugs manufacturing is a historical issue affecting varied countries worldwide. In the sixties the main drugs for recreational use included cocaine, heroin, amphetamines and LSD; but during the seventies and eighties this issue escalated with the advent of the "designer drugs". A new class of chemical substances produced in underground laboratories, which offered an easier and cost-effective alternative to heroin production [1]. More recently, the fingerprints of lethal opioid overdoses has shown an interesting change over the last twenty-four months, namely in areas of Rhode Island and Pennsylvania $[2,3]$, where approximately sixty-four deaths were recorded related to a new class of "designer" opioids that according to some news reports may be migrating further into the southeast [2]. The 4-phenylpiperidines, a cohort of synthetic opioids, from which these "designer narcotics" are derived, are related to a common anesthetic drug, known as fentanyl [2, 4]. They are related to a common anesthetic drug, known as fentanyl. The latter acts as a robust $\mu$-opioid receptor agonist within the central nervous system (CNS) causing exhilaration, respiratory depression and analgesia[4]. In the black market world, a spectrum of synthetic opioid derivates have been identified as "China White", named after their presumed purity [5]. Several official sources have equated this nomination to anecdotal evidence obtained from various reputable forums related to the use and handling of these synthetic opioids revealing that "China White" also refers to other members of the 4-phenylpiperidines. These include p-fluorofentanyl, $\alpha$-methylfentanyl, and at times heroin, also is thought to be "pure" [2]. It appears that the name "China White," alludes to the purity of the substance more so than to its factual identity according to information noted in several of these subject forums [2]. However, considering the variability of compounds that may exist in a given sample, the inconsistency of dosages, and their potency (typically ranging from fifteen to three-hundred times the potency of morphine [6]); emergency rooms (ER) and intensive care units (ICU) may be facing a series of serious considerations in terms of the diagnosis and management of these new realm of modern-day opioid overdoses.

\section{Background}

Clinically, the classic teaching for the identification of isolated opioid overdose proposes a specific triad; which consists of (a) decreased respiratory drive as determined by a respiratory rate of less than twelve breaths per minute, (b) pupillary constriction on eye exam, and (c) altered mental 
status. Altogether, these clinical manifestations are commonly known as the "heroin overdose syndrome" [7]. Once identified, naloxone, a strong $\mu$-opioid receptor competitive antagonist $[8,9]$, is administered as first-line therapy in progressive empiric dosing to achieve normalization of the respiratory rate and mental status [10], followed by the appropriate disposition based on global clinical assessment and response. This is followed by monitoring for four to six hours after clinical improvement is noted. Subsequent monitoring of secondary complications such as rhabdomyolysis, prolonged hypoxemia, myoglobinuric kidney failure, and hypothermia is frequently recommended in consideration for high-risk populations such as the opioid dependent and the elderly [11].

Evidently, modern-day designer opioid formulations pose potential challenges for acute care clinicians, primarily due to the lack of information on the exact constituents within these illicit substances. Furthermore, the experimental findings from animal models warrant improved diagnosis, treatment, monitoring, and reporting methods for suspected designer opioid toxicities [12].

\section{Designer Opioid Facts}

A health alert issued by the Centers for Disease Control (CDC) in 2013 reported fourteen deaths between the ages of nineteen and fifty-seven attributed to the use of acetyl-fentanyl in the state of Rhode Island [2, 13]; fifty of these cases were reported in Pennsylvania [3]. "Designer" opioids elaboration commonly involve the use of other substances (i.e. heroin), which often act as surplus adulterants, and may further complicate the medical scenario and clinical manifestations [14]. Both, formal literature and anecdotal reviews indicate, that acetyl-fentanyl chemical mixtures may include heroin constituents. Furthermore, the drug could be potentially packed as pills and sold on streets as oxycodone [14]. However, the most common form of the substance is powder, typically found in containers labeled "not for human use" or "experimental"; thereby it can be easily accessed from a socio-legal standpoint.

\section{Pharmacokinetics}

Related to fentanyl, 4-phenylpiperidines such as $\alpha$-methylfentanyl, p-fluorofentanyl, and the iconic acetylfentanyl, are all thought to be products of contamination during key steps of fentanyl synthesis [15]. As such, the clandestine synthesis of any synthetic opioid should be suspected to have some proportion of each in the context of an acute intoxication, namely if any historical findings suggest the use of "China White" or other related narcotics. Additional findings that should prompt a similar suspicion are the presentation for acute opioid overdose in which the container is labeled as discussed previously, or in any local community where 4-phenylpiperidine synthesis represents a social concern. Fentanyl analgesic effects are known to be approximately eighty times the strength of morphine as determined by ED50 in clinical studies [6]. Compared to morphine, the popular acetyl-fentanyl is approximately five times stronger. However, $\alpha$-methylfentanyl is nearly three-hundred times the potency of morphine. It should also be noted that given the variable affinity of these mixed compounds, a half-life is difficult to predict for multiple reasons. These include systemic distribution, hepatic function, renal function, GI transit (if taken orally), adulterants presence, and receptors saturation, among others [11]. Among the narcotic formulations, which exhibit highly predictable onset of effects, duration, and excretion times; naloxone has a shorter half-life compared to other drugs of ingestion. Thus, after the effects of naloxone begin to regress, a relapse of symptoms may take place in the setting of a longer-acting narcotic, as may be seen with 4-phenylpiperidine with slower onset of symptoms [11].

\section{Diagnosis and Treatment}

The classic triad of pupillary constriction, altered mental status, and respiratory depression may often prompt the clinical suspicion of opioid toxicity. Moreover, given the propensity for many of these substances to be used as part of a greater comorbid paradigm (i.e., multidrug use), with the presence of adulterant substances [14], or in altered physiological stage (e.g., opioid dependent and elderly patients), pupillary constriction and mental status can often be misleading if absent [11]. Nevertheless, few substances other than opioids actually cause significant respiratory depression (to a rate of less than twelve), representing a more reliable manifestation of whether or not narcotics are a likely enough culprit to treat empirically for acute opioid intoxication [11].

Conventional treatment guidelines for opioid intoxication indicate that an initial dose of naloxone $(0.04 \mathrm{mg})$ must be administered, while searching for a notable increase in the respiratory rate of the patient. Which should be evident 2-3 minutes after the initial dose is administered. Thereafter, increasing doses of $0.5 \mathrm{mg}$ should be attempted if no response, followed by $2 \mathrm{mg}, 4 \mathrm{mg}, 10 \mathrm{mg}$, and $15 \mathrm{mg}$, respectively; until an increase in the ventilation rate is recognized. This establishes the responsiveness to the naloxone treatment protocol. At times, subsequent monitoring and repeated administrations may be suitable, including formal admission to ICU [11].

If intoxication with any other opioid is suspected, the treatment should proceed in accordance to aforementioned guidelines. That includes a complete and continuous clinical care as well. On the other hand, if "designer" opioids, "China White" or other "synthetic heroin" intoxication is suspected, current reports posit that many of these 4-phenylpiperidine molecules test "positive" for fentanyl on ELISA screening, but are not noted to be fentanyl on Gas Chromatography (GC) / mass spectrometry (MS) [16]. Therefore, CDC 
recommendations establish that ELISA screening should be obtained, and if positive, GC/MS may be performed for clinical assurance and validation [2, 3]. Thus, the recommendations per this communication are to treat suspected intoxications according to the established guidelines, paying close attention to the documentation of specific clinical progressions. In cases of "designer" opioids, consider screening with ELISA assay for fentanyl, and if positive, pursue confirmatory testing with GC/MS to identify culprit substances that may correlate with time improvements after naloxone therapy; and time of relapse symptoms for the optimization of the clinical decision-making observational periods and monitoring practices.

\section{Summary}

We are living in times of much fortune; it manifests in different forms. These include, wealth, sovereignty, freedom, and perhaps even the limitless access to the internet resources is one of the major manifestations of our media extravaganza. Ironically, these web-based means contain all sorts of information, some of which offer a direct path to misfortune through the dissemination of material for the production of illicit synthetic substances or "designer" drugs $[1,14]$. Not too savvy, in an era where addictive disorders represent one of the world's major socioeconomic burdens, as they embody diseases, criminality and death [17]. Meanwhile, the death cases associated to these so-called "designer" drug practices are progressing almost inaudibly $[2,3,13]$. But, in the medical arena proactive clinical considerations ought to be taken when suspected opioid intoxication cases are encountered [18].

In most cases, a clinical suspicion may suffice to pursue empiric treatment with naloxone considering that there are no significant side effects to its use in such circumstances. This treatment protocol should proceed as recommended by the established treatment guidelines for opioid intoxication [11]. Though it is suggested that substance identification be pursued for what may eventually lead to more directed clinical decision-making with guided monitoring practices when potential intoxication cases are encountered. Initially, GC/MS may not be a practical option in a setting of acute opioid intoxication; however ELISA immunoassay screening is suggested based on anticipatory clinical suspicion $[2,3,16]$. In addition, it would be interesting to correlate the clinical progressions with both, naloxone regimen and "time to recovery" data, as they may play a significant role in the monitoring recommendations and the length of treatment when "designer" opioid use is suspected. In the context of added agents by users, there are other considerations such as fungemias, commonly acquired from the use of lemon juice as a liquefying agent, and other blood-related infections related to "cutting" agents that may be of therapeutic interest to treat patients with further clinical manifestations. These may include but are not limited to sepsis, hepatic failure, active/ongoing HIV/AIDS, or renal failure in the setting of secondary rhabdomyolysis. Altogether, these conditions have been widely documented in the literature regarding illicit intravenous drug use; and should be carefully considered when clinical opioid toxicity crosses paths with other acute clinical syndromes.

Drug addiction is a terrible disease of modern society that warrants continued scientific attention, laws, and policy reform. The information presented herein adds to the growing body of evidence on drug addiction epidemics. Although opioid "designer" drugs are not currently a major addiction concern, it can potentially add to the existent substance dependence disorders chronicle, particularly if the clandestine heroin supplies decline. Proactively, drug addiction scientists, epidemiologists and physicians, ought to continue collaborating in more research investigations to deliver new insights for a better understanding of the addictive disorders, improved treatments with clinically sustainable options, but more importantly for the conveyance of a potential cure.

\section{REFERENCES}

[1] F.I. Carroll, A.H. Lewin, S.W. Mascarella, H.H. Seltzman, P.A. Reddy, Designer drugs: a medicinal chemistry perspective, Annals of the New York Academy of Sciences, 1248 (2012) 18-38.

[2] C. Centers for Disease, Prevention, Acetyl fentanyl overdose fatalities--Rhode Island, March-May 2013, MMWR. Morbidity and mortality weekly report, 62 (2013) 703-704.

[3] ProDajA. Programs, Department of Drug and Alcohol Programs warns about acetyl fentanyl: drug caused at least 50 fatalities this year in Pennsylvania.

[4] B.T. Wang, J.M. Colby, A.H. Wu, K.L. Lynch, Cross-Reactivity of Acetylfentanyl and Risperidone With a Fentanyl Immunoassay, J Anal Toxicol, (2014) 672-675.

[5] G.L. Sternbach, J. Varon, 'Designer drugs'. Recognizing and managing their toxic effects, Postgraduate medicine, 91 (1992) 169-171, 175-166.

[6] D.E.A.-D.o.D. Control, Acetylfentanyl (N-(1-phenethylpiperidin-4-yl)-N-phenylacetamide), (2013).

[7] K.A. Sporer, Acute Heroin Overdose, Annals of Internal Medicine, 130 (1999) 584-590.

[8] A.M. Bailey, D.P. Wermeling, Naloxone for opioid overdose prevention: pharmacists' role in community-based practice settings, The Annals of pharmacotherapy, 48 (2014) 601-606.

[9] J.M. Chamberlain, B.L. Klein, A comprehensive review of naloxone for the emergency physician, The American journal of emergency medicine, 12 (1994) 650-660.

[10] D. Belz, J. Lieb, T. Rea, M.S. Eisenberg, Naloxone use in a tiered-response emergency medical services system, Prehospital emergency care : official journal of the National Association of EMS Physicians and the National Association 
of State EMS Directors, 10 (2006) 468-471.

[11] E.W. Boyer, Management of Opioid Analgesic Overdose, New England Journal of Medicine, 367 (2012) 146-155.

[12] M. Ford, R.S. Hoffman, L.R. Goldfrank, Opioids and designer drugs, Emergency medicine clinics of North America, 8 (1990) 495-511.

[13] M.C. Mercado-Crespo, S.A. Sumner, M.B. Spelke, D.E. Sugerman, C. Stanley, Notes from the field: increase in fentanyl-related overdose deaths - Rhode Island, November 2013-March 2014, MMWR. Morbidity and mortality weekly report, 63 (2014) 531.

[14] J.M. Stogner, The potential threat of acetyl fentanyl: legal issues, contaminated heroin, and acetyl fentanyl "disguised" as other opioids, Annals of emergency medicine, 64 (2014) 637-639.

[15] W.H. Soine, Clandestine drug synthesis, Medicinal research reviews, 6 (1986) 41-74.

[16] G.L. Henderson, M.R. Harkey, A.D. Jones, Rapid screening of fentanyl (China White) powder samples by solid-phase radioimmunoassay, J Anal Toxicol, 14 (1990) 172-175.

[17] M.D. Stein, Medical consequences of substance abuse, The Psychiatric clinics of North America, 22 (1999) 351-370.

[18] M. Martin, J. Hecker, R. Clark, J. Frye, D. Jehle, E.J. Lucid, F. Harchelroad, China White epidemic: an eastern United States emergency department experience, Annals of emergency medicine, 20 (1991) 158-164. 